\title{
Sibling astrocytes share preferential coupling via gap junctions
}

\author{
Yolanda Gutiérrez $^{1 *}$ | Jorge García-Marques ${ }^{1 *}$ | Xinhe Liu' ${ }^{2}$ | Lluis Fortes-Marco ${ }^{1}$ | \\ Rebeca Sánchez-González $^{1}$ | Christian Giaume ${ }^{2}$ | Laura López-Mascaraque ${ }^{1}$ (]
}

${ }^{1}$ Instituto Cajal-CSIC, Molecular, Cellular and Developmental Neurobiology Department,

Madrid, Spain

${ }^{2}$ Center for Interdisciplinary Research in Biology (CIRB), Collège de France, Paris, France

\section{Correspondence}

Laura López-Mascaraque, Instituto Cajal-CSIC, Molecular, Cellular and Developmental Neurobiology Department, Madrid, Spain. Email: mascaraque@cajal.csic.es

Funding information

Ministerio de Economía y Competitividad, Grant/Award Number: MINECO;

BFU2016-75207-R

\begin{abstract}
Astrocytes are organized as communicating cellular networks where each cell is connected to others via gap junctions. These connections are not pervasive and there is evidence for the existence of subgroups composed by preferentially connected cells. Despite being unclear how these are established, we hypothesized lineage might contribute to the establishment of these subgroups. To characterize the functional coupling of clonally related astrocytes, we performed intracellular dye injections in clones of astrocytes labeled with the StarTrack method. This methodology revealed sibling astrocytes are preferentially connected when compared to other surrounding astrocytes. These results suggest the role of the developmental origin in the organization of astrocytes as intercellular networks.
\end{abstract}

\section{KEYWORDS}

astrocyte, clone, connectivity, coupling, IUE, ontogeny

\section{1 | INTRODUCTION}

Astrocytes play a myriad of fundamental roles in the central nervous system, such as providing structural and metabolic support or controlling the blood flow (Attwell et al., 2010; Ballabh, Braun, \& Nedergaard, 2004). A prominent feature of astrocytes is that they establish intercellular networks through gap junctions (Orthmann-Murphy, Abrams, \& Scherer, 2008). In the brain, astrocytes express the highest expression of connexins (Cxs), the protein constituents of gap junction channels that provide the molecular basis for direct cell-to-cell communication (Giaume, Koulakoff, Roux, Holcman, \& Rouach, 2010). These channels allow the exchange of ions and small signaling molecules between connected cells. Two main Cxs are expressed in astrocytes: Cx43, which is already present at birth, and $\mathrm{C} \times 30$, which is detected by the beginning of the second postnatal week (Nagy \& Rash, 2000). Gap junctional communication (GJC) is known to contribute to a range of important functions of astrocytes including spatial buffering, propagation of intercellular calcium waves, and trafficking of metabolites among others (Giaume et al., 2010).

*Yolanda Gutiérrez and Jorge García-Marques contributed equally to this work.
Although astrocytes were initially thought to form a pervasive glial syncitium, there is now evidence for a more specialized network organization (Claus et al., 2018; Houades et al., 2008; Roux, Benchenane, Rothstein, Bonvento, \& Giaume, 2011). Such specialization was observed after injecting an intracellular dye into single astrocytes, which revealed unlabeled astrocytes located within the perimeter of dye diffusion. This is particularly interesting in regions where the anatomical organization strikingly reflects the circuit function, including the barrel cortex (Houades et al., 2008), the olfactory glomeruli (Roux et al., 2011) and the barreloid field in the thalamus (Claus et al., 2018). Similarly, other regions such as hippocampus exhibited a certain degree of specificity in GJC between astrocytes (Houades et al., 2006).

The mechanism underlying this preferential coupling is yet to be investigated. The fact that subpopulations of astrocytes can be distinguished based on their morphologic, molecular and functional properties (Oberheim, Goldman, \& Nedergaard, 2012; Theis \& Giaume, 2012), suggests that astrocyte heterogeneity could sustain networks of preferential coupling. Such heterogeneity is established during development as different types of astrocytes emerge from independent progenitor cells (García-Marqués \& López-Mascaraque, 2013; Hochstim, Deneen, Lukaszewicz, Zhou, \& Anderson, 2008). Therefore, we hypothesized that cell lineage could determine preferential 
intercellular coupling between sibling astrocytes, as occurs with neurons (Yu et al., 2012). To investigate this hypothesis, we applied the StarTrack method (García-Marqués \& López-Mascaraque, 2013) to label astrocytes clones derived from single progenitors. Next, we performed intracellular dye injections in acute brain slices and analyzed the labeling pattern in clonally related or unrelated astrocytes. Such approach showed that astrocytes are organized as heterogeneously coupled networks based on their ontogenic origin.

\section{2 | MATERIALS AND METHODS}

\section{1 | Animals}

Wild type C57BL/6 mice of either sex were raised at the Cajal Institute animal facility. All the experiments complied with the ethical regulations on the use and welfare of experimental animals of the European Union (2010/63/EU) and the Spanish Ministry of Agriculture (RD 1201/2005 and L 32/2007) and all procedures approved by the Cajal Institute, CSIC Animal Experimentation Ethics Committees, and the Community of Madrid (Ref. PROEX 44/14). Day of vaginal plug detection was defined as the first embryonic Day 0 (EO) and the day of birth was defined as postnatal day 0 (PO).

\subsection{StarTrack plasmids}

StarTrack plasmids were used as previously described (García-Marqués \& López-Mascaraque, 2013). Briefly, the expression of four fluorescent reporters was driven by the human glial fibrillary acidic protein (hGFAP) promoter, thus confining their expression to astrocytic lineages. The fluorescent proteins chosen for this study have been enhanced green fluorescent protein (EGFP), mTSapphire, mCerulean, and yellow fluorescent protein (YFP). In order to achieve a higher combinatorial probability, nuclear forms of these markers were also used. To ensure the nuclear expression, XFPs are fused to the human $\mathrm{H} 2 \mathrm{~B}$ histone. Due to the multiple cellular divisions astrocyte precursors undergo, the genomic integration of the fluorescent reporters is needed to ensure an inheritable mark. This was achieved by inclusion of the hGFAP-XFP sequences between terminal repeats of the PiggyBac transposon and subsequent co-expression with a plasmid encoding the hyperactive PiggyBac transposase (hyPBase) under the ubiquitous $\mathrm{CMV}$ promoter.

\section{3 | In utero electroporation (IUE)}

Pregnant mice at embryonic day 14 (E14) were anesthetized by isofluorane inhalation (Isova vet, Centauro, $2 \mathrm{~mL} / \mathrm{L}$ ) and maintained at $37^{\circ} \mathrm{C}$. Antibiotic enrofloxacine $(5 \mathrm{mg} / \mathrm{kg}$, Baytril Bayer) and antiinflammatory/analgesic meloxicam $(300 \mu \mathrm{g} / \mathrm{kg}$, VITA Laboratories) were subcutaneously administered before the surgery. Uterine horns were exposed by a midline incision through the skin and the abdominal wall. The uterus was trans-illuminated with cold light and $2 \mu \mathrm{L}$ of the plasmid mixture (2-5 $\mu \mathrm{g} \mathrm{DNA} / \mu \mathrm{L}$ containing $0.1 \%$ fast green) was injected into the lateral ventricles (LV) of E14 embryos using a pulled glass micropipette attached to a microinjection system. Then, each embryo was held between $3 \mathrm{~mm}$ tweezer-type electrodes (Sonidel) to deliver one train of 5 square wave pulses $(50 \mathrm{~ms}$ and $35 \mathrm{~V}$ each, followed by $950 \mathrm{~ms}$ intervals). In all cases, the electroporated region was the ventricular zone/subventricular zone in the dorso-lateral area. After electroporation, the uterus was repositioned and the abdominal cavity was sutured. Mice were allowed to recover and embryos continued development until birth.

\subsection{Patch-clamp recording and dye coupling of astrocytes}

Experiments were carried out in acute cortical brain slices prepared from mice that show expression of fluorescent reporters directed by the StarTrack mixture. Mice were aged between P15 and P30. Coronal brain slices (300 $\mu \mathrm{m}$ thick) containing the somatosensory cortex were cut using a vibratome (Microm HM $650 \mathrm{~V}$, Walldorf, Germany) filled with ice-cold artificial cutting solution containing (in mM) 222 sucrose, $27 \mathrm{NaHCO}_{3}, 2.6 \mathrm{KCl}, 1.25 \mathrm{NaH}_{2} \mathrm{PO}_{4}, 2 \mathrm{CaCl}_{2}$, $7 \mathrm{MgSO}_{4}$, and 0,1 ascorbic acid. They were incubated at $32^{\circ} \mathrm{C}$ for $30 \mathrm{~min}$ in artificial cerebrospinal fluid (ACSF) containing (in $\mathrm{mM}$ ) $125 \mathrm{NaCl}, 2.5 \mathrm{KCl}, 25$ glucose, $25 \mathrm{NaHCO}_{3}, 1.25 \mathrm{NaH}_{2} \mathrm{PO}_{4}, 2 \mathrm{CaCl}_{2}$, and $1 \mathrm{MgCl}_{2}$, continuously aerated with $95 \% \mathrm{O}_{2} / 5 \% \mathrm{CO}_{2}(\mathrm{pH} 7.4)$. Then slices were transferred at room temperature $\left(20-22^{\circ} \mathrm{C}\right)$ before use. The slices were placed in a submerged recording chamber superfused with aerated ACSF at a rate of $2 \mathrm{~mL} / \mathrm{min}$ and observed using an up-right fixed stage microscope (Axioskop FS; Zeiss, Oberkochen, Germany) equipped with Nomarski optics and an infrared video camera (Newvicon C2400; Hamamatsu, Shizuoka, Japan). Clones of fluorescent astrocytes were identified with epifluorescence and whole-cell patch-clamp recording of astrocytes was performed with pipettes (3-8 $\mathrm{M} \Omega$ ) filled with internal solution that contains (in mM) $105 \mathrm{~K}$-Gluconate, $30 \mathrm{KCl}, 10$ Hepes, 10 Phospho-Creatine Tris, 4 ATP- $\mathrm{Mg}^{2+}, 0.3$ GTP-Tris, and 0.3 EGTA (adjusted to $\mathrm{pH} 7.4$ with $\mathrm{KOH}$ ). Experiments were conducted at room temperature (20 to $22^{\circ} \mathrm{C}$ ). Membrane voltages and currents were amplified by a MultiClamp 700B amplifier, sampled by a DIGIDATA 1322A Interface, and patch-clamp recordings $(5 \mathrm{kHz}$ sampling and $3 \mathrm{kHz}$ filtering) were performed with Pclamp9 software (Axon Instruments, Foster City, CA). Series resistances were compensated at $80 \%$. Input resistance (Rin) was measured in voltage-clamp mode by applying hyperpolarizing voltage pulses $(10 \mathrm{mV}, 150 \mathrm{~ms}$ ) from a holding potential of $-80 \mathrm{mV}$. To assess the level of GJC, dye coupling experiments were performed by adding sulforhodamine B (1 mg/mL; Molecular Probes, Eugene OR) to the pipette solution before patch-clamp recording. Recorded cells were loaded with the dye for $20 \mathrm{~min}$ in the whole-cell configuration and current-clamp mode to allow its diffusion among cells connected by GJCs. Brain slices were subsequently fixed in $4 \%$ paraformaldehyde and $4 \%$ sucrose in PBS and, after PBS washes, mounted in Mowiol on glass microscope slides for imaging. 


\section{5 | Image acquisition}

Images of the fixed slices were acquired on a Leica TCS-SP5 confocal microscope, acquiring one or two different channels simultaneously. The excitation (Ex) and emission (Em) conditions for each fluorophore and dye were (in nanometers): mT-Sapphire (Ex: 405; Em: 525-553), mCerulean (Ex: 458; Em: 464-481), EGFP (Ex: 488; Em: 496-526), YFP (Ex: 514; Em: 520-543), sulforhodamine B (Ex: 561; Em: 585-625). Each channel was assigned a color corresponding to their emission color, except for mT-Sapphire, which was labeled as dark blue. Maximum projection images of the z-stacks were generated using the confocal software (Leica LAS AF Software). The projections for each channel were adjusted uniformly and overlaid using the Adobe Photoshop CS5 software. Previous experiments (Figueres-Oñate, García-Marqués, \& López-Mascaraque 2016; García-Marqués \& López-Mascaraque, 2013) allowed the definition of the spectral windows and confocal acquisition parameters for optimal fluorescence detection without overlapping of the channels.

\section{6 | Image analysis and data processing}

The image software Image J v1.41 (NIH) was used to measure the sulforhodamine B mean intensity of the labeled cells. Only the fluorescence intensity at the somas was analyzed and experimental conditions and image acquisition parameters were maintained across experiments. Distances were measured using the TrackEM2 Fiji plugin and performed from the center of the soma of the injected cell to the center of the soma of the labeled cell, across the XYZ space. Patched astrocyte was not included in any of the quantifications, neither distance nor intensity. (a)

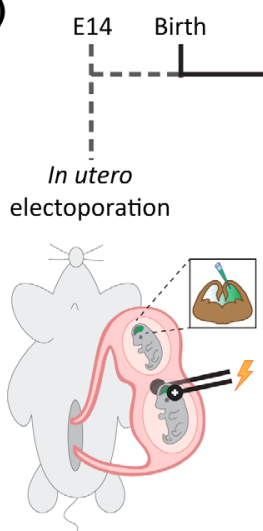

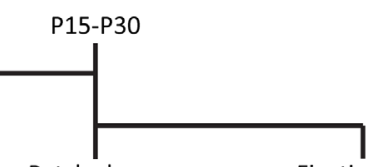

Patch clamp and dye coupling

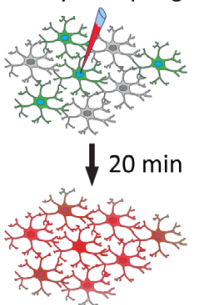

Fixation and image acquisition

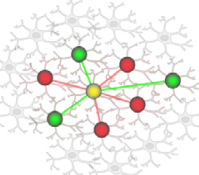

(b)

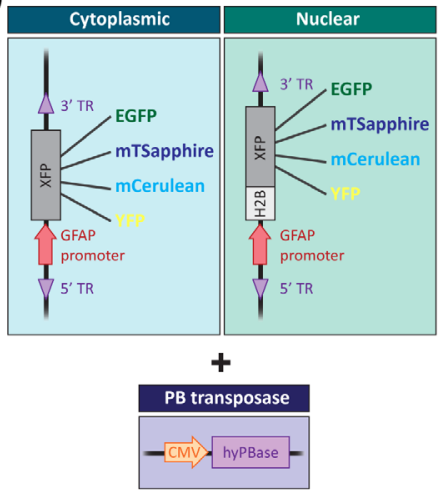

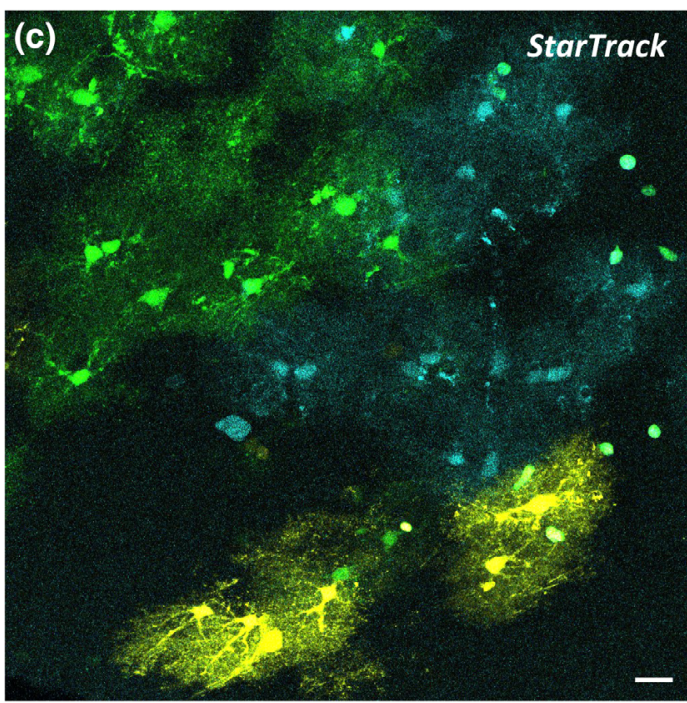

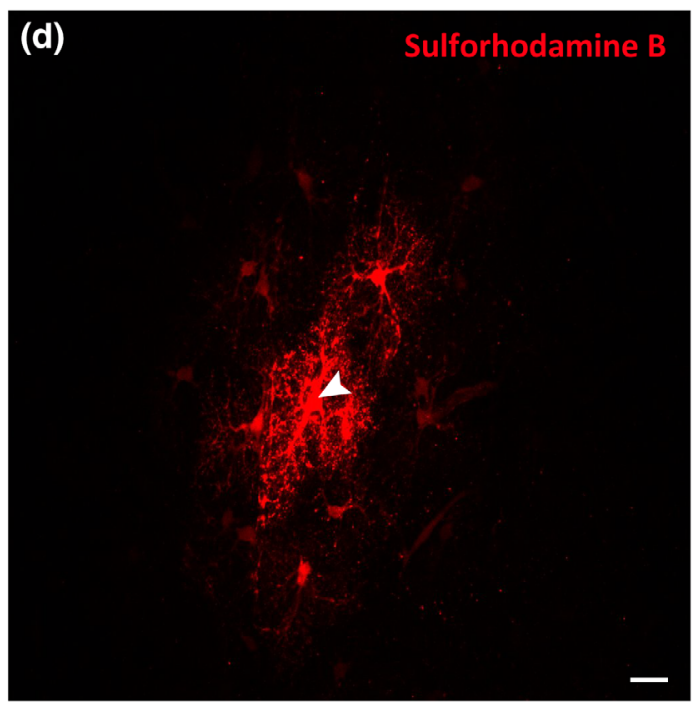

FIGURE 1 Methodology overview. (a) At embryonic Day 14 (E14) embryos were electroporated after ventricular injection of the plasmid mixture. Embryos continued to grow between postnatal Days 15 and 30 (P15-P30) mice were sacrificed and acute cortical slices were obtained. Fluorescent visualization of the cortical glial clones allowed whole-cell patch clamping of individual StarTrack-labeled astrocytes. The recording pipette contained a fluorescent dye sulforhodamine B, which was allowed to diffuse among connected astrocytes for 20 min. Analysis of the fluorescence intensity and diffusion radius of the labeling was performed after fixation of the injected slices and posterior image acquisition. (b) A reduced StarTrack plasmid mixture used to label unique clones of astrocytes while allowing sulforhodamine B imaging. (c, d) Representative images of StarTrack-labeled astrocyte clones, (c) and sulforhodamine B diffusion (d) from a single injected astrocyte (arrowhead) in an acute cortical slice. Scale bars $20 \mu \mathrm{m}$ 
Statistical analysis of the data and graphical representations were performed using R statistical software version 3.5 (R Core Team, 2018).

\section{3 | RESULTS}

\subsection{Simultaneous assessment of lineage and astroglial networks}

The StarTrack approach allows labeling of each astrocyte clone with a unique color code in the adult mouse brain (García-Marqués \& LópezMascaraque, 2013). This is based on the generation of a stable colorcode in a single progenitor cell that is inherited by its progenies. To create this code, a mixture of different reporters under the regulation of the hGFAP promoter is transfected by intrauterine electroporation (IUE). Flanked by the inverted repeats of the PB transposon, these reporters are integrated into the genome by cotransfection with a hyPBase transposase plasmid. Once a specific combination of reporters is integrated, this mark remains stable through cell divisions, whereas nonintegrated plasmids are diluted and do not interfere with the analysis (García-Marqués \& López-Mascaraque, 2013) (Figure 1). In this study, a reduced StarTrack mix was used, which allowed the combination of this method with the intracellular injection of sulphorodamine B. Since astrocytes are locally divided and occupy specific spatial domains (Bushong, Martone, Jones, \& Ellisman, 2002; Ogata \& Kosaka, 2002), the use of this reduced StarTrack method combined with the analysis of small cortical subfields results in clear identification of astrocyte clones, defined as those astrocytes derived from single progenitors. The possible combinations of the eight fluorescent reporters (nuclear and cytoplasmic) are sufficient to determine clonal relationships, while at the same time they allow the use of red and far-red channels for subsequent analysis of gap junctional coupling and dye spread.

\section{2 | Sibling astrocytes exhibit preferential coupling}

Following the optimization of the experimental settings for the combined analysis, we set out to evaluate how clones of astrocytes are connected via gap junctions. First, we electroporate a reduced version of StarTrack at E14 (Figure 1b). At postnatal Day 15-30 (P15-P30) we performed dye-coupling experiments in acute brain slices from electroporated mice. Sulforhodamine B, a gap junction channel permeable dye, was injected into a single StarTrack-labeled astrocyte and it was allowed to passively diffuse for 20 min (Figure 1a). This fluorescent dye can be easily detected under $565 \mathrm{~nm}$ light and it allows the visualization of astroglial networks (Giaume, Orellana, Abudara, \& Sáez, 2012). Twenty-four independent clones/brain slices were analyzed, and following fixation of the slices, confocal images were acquired to assess the clonal labeling and the dye coupling within astroglial networking (Figure 1c,d; Figure 2).

A total of 142 sulforhodamine B-labeled cells, both clonally and non-clonally related to the patched astrocyte, were analyzed. For all
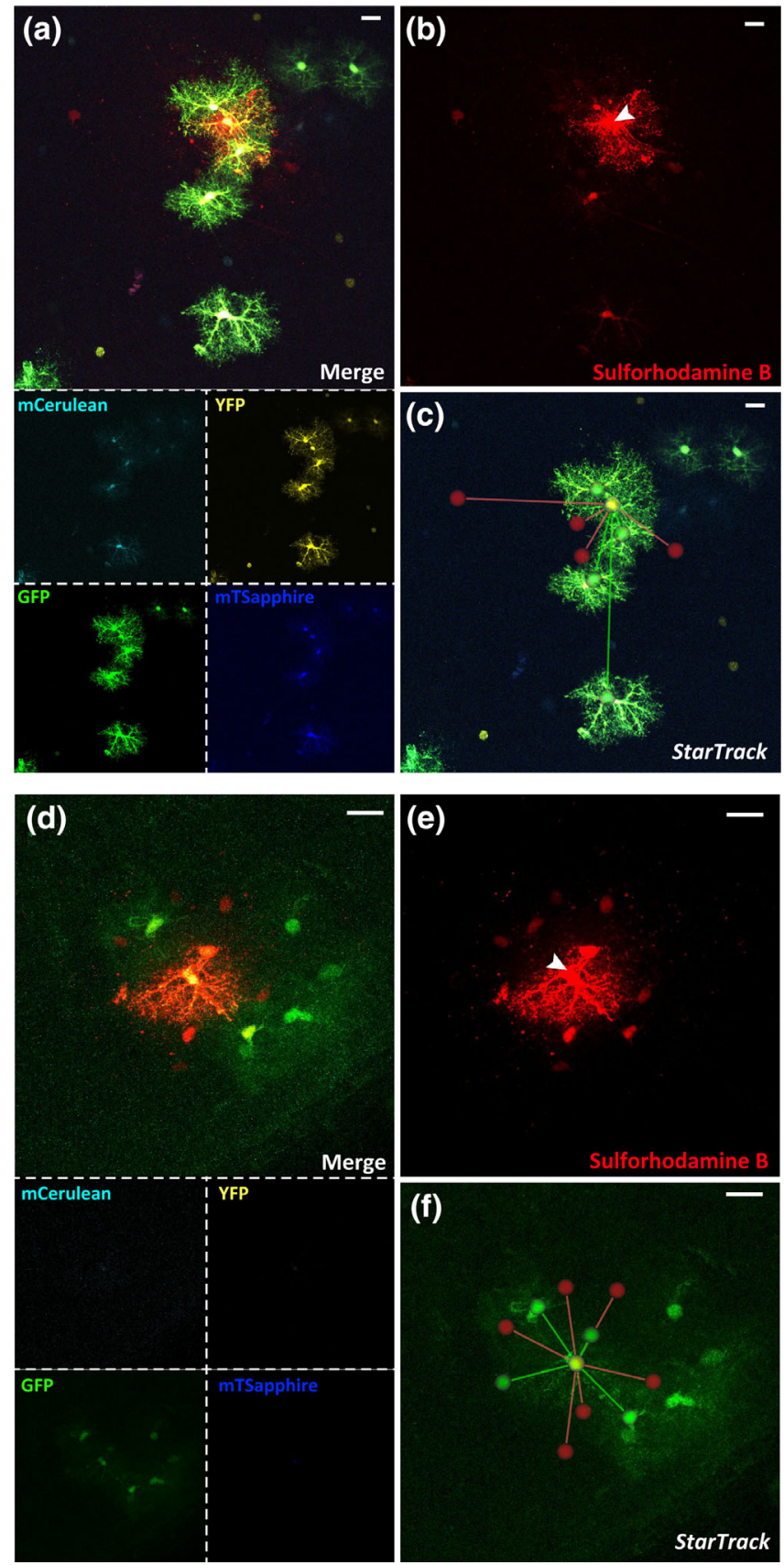

FIGURE 2 Representative images of sulforhodamine B signal distribution and StarTrack labeling of sibling astrocytes. (a, d)

Merged images of the Z-stack projection for all acquired channels showing both the clonal labeling and Sulforhodamine B dye diffusion. Insets below show individual channels of the StarTrack labeling for clonal analysis (mCerulean, YFP, EGFP, and mT-Sapphire). (b, e) Projection images showing sulforhodamine B labeling obtained from the diffusion after injection of a single astrocyte (arrowheads). Images correspond to $a$ and b, respectively. (c, f) Stick-and-ball models corresponding to all astrocytes showing sulphorhodamine B labeling are overlaid on the merged projection images from the StarTrack channels. Cells corresponding to the clone of the injected one are shown in red green, whereas cells from a different clone are displayed in red. Patched astrocytes are shown in yellow. Scale bars $20 \mu \mathrm{m}$ 

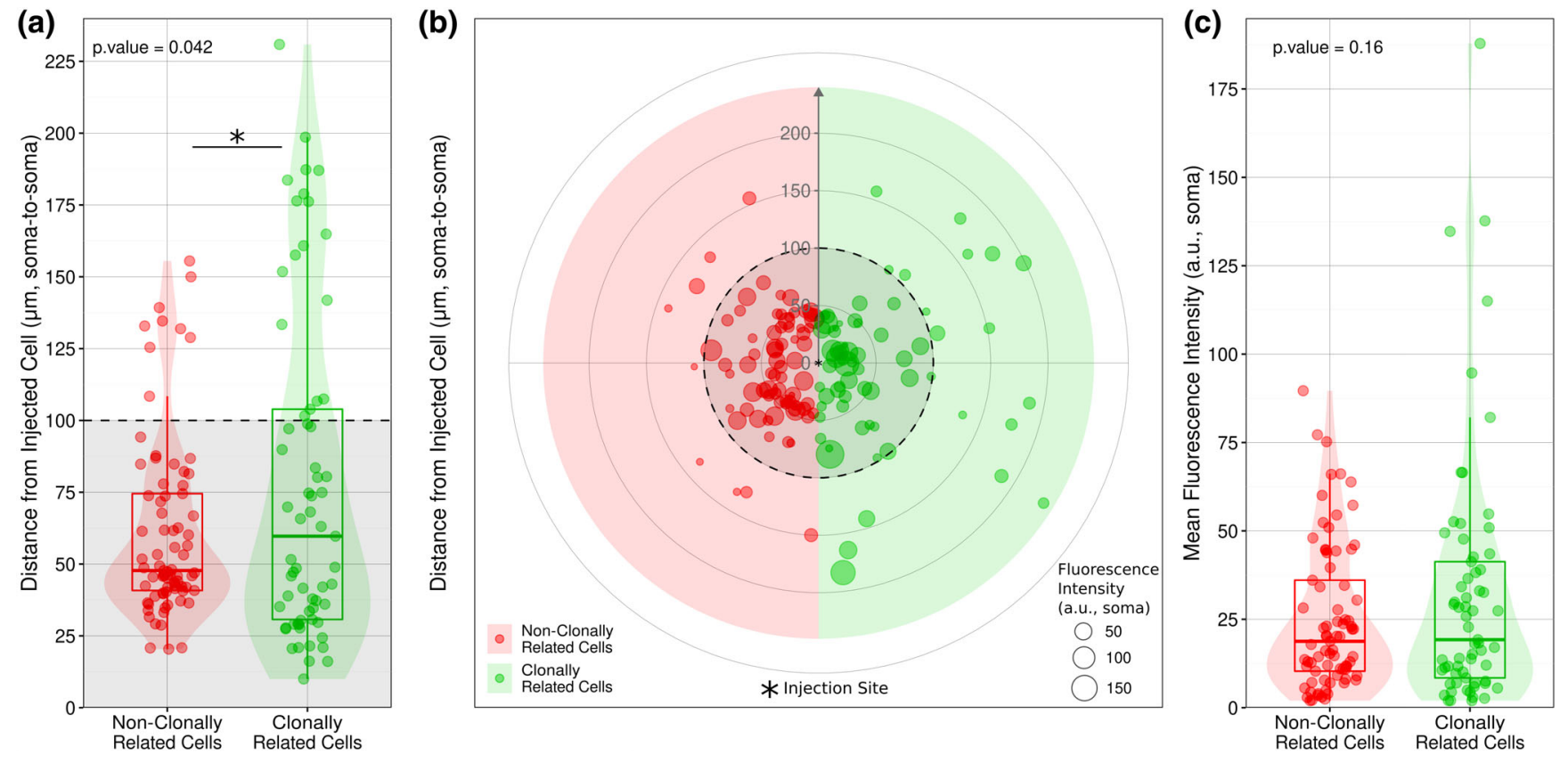

FIGURE 3 Sibling astrocytes exhibit preferential coupling. Statistical data analyses of clonally- and non-clonally-related astrocytes from 24 independent experiments with a total of 142 sulforhodamine B-labeled cells. (a) Violin and box plots of the distance from the soma of the patched astrocyte to the center of the individual sulforhodamine B labeled soma of clonally- and non-clonally related astrocytes. The distance of dye diffusion is significantly higher in the clonally related cells (green, $p$-value $=.042$, $t$-test) compared to the non-clonally related (red). Shaded area (dashed line), shows the range of passive dye diffusion process, previously reported at $\sim 100 \mu \mathrm{m}$ (Houades, Koulakoff, Ezan, Seif, $\&$ Giaume, 2008). Most cells observed at larger distances correspond to astrocytes clonally-related to the patched cell. (b) Radar plot of individual cell distribution along distance for non-clonally (red) and clonally related astrocytes (green). Point size indicates sulforhodamine B labeling intensity of the soma in arbitrary units (a.u.). (c) Violin and box plots of the intensity of clonally (green) and non-clonally (red) related astrocytes. Mean intensity is not significantly different between both conditions ( $p$-value $=.16, t$-test)

cells, we measured both the distance from the center of the soma of the patched astrocyte to the center of the soma of the analyzed cells (Figure 3a,b). Moreover, sulforhodamine B intensity at the soma of the analyzed astrocytes was also measured (Figure 3b,c). A t-test analysis revealed a significant difference $(p$-value $=.042)$ in the distance of diffusion between non-clonally related (Figure $3 a$, red) and clonally related cells (Figure 3a, green). Previous studies analyzing a similar purely passive dye diffusion process have reported a maximum distance of dye presence at $\sim 100 \mu \mathrm{m}$ (Houades et al., 2008). Our data show that the majority of cells observed at larger distances correspond to astrocytes clonally related to the patched cell (Figure 3a,b, dashed lines). Among non-clonally related astrocytes, dye diffusion followed the expected pattern of passive diffusion along a chemical gradient, where the concentration of sulforhodamine B (estimated as fluorescence intensity) depended upon the distance from the dye injection site.

The clonally related cells not only were more broadly distributed (Figure 3a,b, S1a, green), but also exhibited a higher intensity than expected from their distance assuming a purely passive diffussion model (Figure 3b, S1a, green). However, although these differences in dye intensity also followed a similar pattern as it was reported for the distance results (Figure 3c, S1a, green), the data analysis failed to prove a statistical significance ( $p$-value $=.16$ ). Therefore, although the intensity/distance ratio (Figure S1b) reveals a higher value for sibling astrocytes when compared to non-clonally related cells, the result was not statistically significant $(p$-value $=.1$ ).

In summary, dye diffusion distance is favored among sibling astrocytes, which exhibit stronger coupling than non-clonally related cells.

\section{4 | DISCUSSION}

Astrocytes have been traditionally considered to play a passive role as supporting cells in the central nervous system. However, mounting evidence suggests their active role in synaptic transmission, information processing and neuronal survival (Allen \& Eroglu, 2017; Verkhratsky \& Nedergaard, 2018). These properties arise from their ability to sense, integrate, and transmit information among them as well as with other cell types such as neurons. In particular, the way astrocytes are connected may affect how they respond to neural activity. The communication among different astrocytes relies on networks of cells coupled via gap junction channels. However, little is known about the mechanisms that astrocytes used to compartmentalize different signals within these networks. Since gap junctions are formed by two hemichannels contributed by each adjacent cell, neighboring astrocytes are more likely to be connected by gap junctions than cells that are far apart (Giaume et al., 2010). However, hemichannels do not always form gap junction channels and have 
been reported to be present in non-contacting membranes of astrocytes to allow intracellular-extracellular communication (Ye, Wyeth, Baltan-Tekkok, \& Ransom, 2003). The variability of intercellular calcium wave propagation observed in experiments of different astrocyte populations (Charles, 1998; Kuga, Sasaki, Takahara, Matsuki, \& Ikegaya, 2011; Sasaki, Kuga, Namiki, Matsuki, \& Ikegaya, 2011; Scemes \& Giaume, 2006) has indeed been suggested to depend not only on the experimental setup but also on heterogeneities in the connections between astrocytes (Scemes \& Giaume, 2006). These heterogeneities have been well characterized for astrocytes in the olfactory bulb, which show preferential gap junctional communication coupling within the confines of glomeruli (Roux et al., 2011). Similar observations have also been reported for astrocytes within somatosensory barrels (Houades et al., 2008) and at the stratum pyramidale of the hippocampus (Rouach, Koulakoff, Abudara, Willecke, \& Giaume, 2008). While it is believed that this peculiar organization could define precise cellular and anatomical domains, neither the functional relevance of such specialized connectivity is known nor how it could ultimately affect astrocytic $\mathrm{Ca}^{2+}$ signaling (Pannasch \& Rouach, 2013).

In the present study, we analyzed for the first time the influence of ontogeny on astrocytic communication and showed that cell lineage determines differences in the extent of coupling. Indeed, we showed that intracellular dye injections into a single astrocyte results in longer passive diffusion distances and higher concentration of dye at the closest distances among sibling astrocytes when compared to nonsibling astrocytes. This better coupling among clonally related astrocytes may be due to the occurrence of more efficient connections, as a result of larger gap junctional plaques, higher conductance, increased fractional opening, and/or open probability of gap junctions channels among sibling astrocytes. Interestingly, a similar preferential coupling between sister excitatory neurons has been described in the developing neocortex (Yu et al., 2012). An exponential curve fitting of the presented dataset would explain the diffusion process and the expected dye intensity decay across distance. However, the goodness-of-fit criteria were not reached (data not shown).

In the olfactory bulb, astrocytes are either preferentially coupled within the same glomerular neuropil or within the interglomerular walls (Roux et al., 2011). Concurrently, astrocyte clones either occupy the glomerular neuropil or the interglomerular walls (García-Marqués \& López-Mascaraque, 2017), as well as how groups of astrocyte clones respond differentially to cortical injury (Martín-López, GarcíaMarques, Núñez-Llaves, \& López-Mascaraque, 2013) or in response to cortical damage induced by a demyelinating EAE-induced mice model (Bribian, Pérez-Cerdá, Matute, \& López-Mascaraque, 2018). Although the clonal distribution has not yet been characterized, other regions such as the barrel cortex (Houades et al., 2008) or the thalamic barreloids (Claus et al., 2018) also exhibit networks of preferentially coupled astrocytes. Therefore, astrocyte clones seem to occupy functional domains and establish preferential coupling within those domains. Given the role of astrocytes in the modulation of neuronal activity (Perea \& Araque, 2007), this organization could have functional consequences such as compartmentalizing this modulatory activity during information processing.
Further experiments and characterization of these observations are needed to assess these hypotheses. Nevertheless, our results already open the door to the existence of another layer of complexity in the brain information processing determined from birth, which may influence, not only in astrocytic communication, but also in regulating neuronal signaling and synaptogenic potential (Allen \& Eroglu, 2017).

\section{ACKNOWLEDGMENTS}

This work was supported by Ministerio de Economía y Competitividad (MINECO; BFU2016-75207-R). We are deeply grateful to Lucila Brocardo for setting up the patch clamp recording, Marta Navarrete for her technical assistance during this research and Oscar Herreras for helpful discussions.

\section{ORCID}

Laura López-Mascaraque (D) https://orcid.org/0000-0002-7154-5687

\section{REFERENCES}

Allen, N. J., \& Eroglu, C. (2017). Cell biology of astrocyte-synapse interactions. Neuron, 96(3), 697-708. https://doi.org/10.1016/j.neuron. 2017.09.056

Attwell, D., Buchan, A. M., Charpak, S., Lauritzen, M., Macvicar, B. A., \& Newman, E. A. (2010). Glial and neuronal control of brain blood flow. Nature, 468, 232-243. https://doi.org/10.1038/nature09613

Ballabh, P., Braun, A., \& Nedergaard, M. (2004). The blood-brain barrier: An overview: Structure, regulation, and clinical implications. Neurobiology of Disease, 16, 1-13.

Bribian, A., Pérez-Cerdá, F., Matute, C., \& López-Mascaraque, L. (2018). Clonal glial response in a multiple sclerosis mouse model. Frontiers in Cellular Neuroscience, 12, 375. https://doi.org/10.3389/fncel.2018. 00375

Bushong, E. A., Martone, M. E., Jones, Y. Z., \& Ellisman, M. H. (2002). Protoplasmic astrocytes in CA1 stratum radiatum occupy separate anatomical domains. The Journal of Neuroscience: The Official Journal of the Society for Neuroscience, 22(1), 183-192.

Charles, A. (1998). Intercellular calcium waves in glia. Glia, 24(1), 39-49.

Claus, L., Philippot, C., Griemsmann, S., Timmermann, T., Jabs, R., Henneberger, C., ... Steinhäuser, C. (2018). Barreloid Borders and Neuronal Activity Shape Panglial Gap Junction-Coupled Networks in the Mouse Thalamus. Cerebral Cortex, 28(1), 213-222. https://doi.org/10. 1093/cercor/bhw368

Figueres-Oñate, M., García-Marqués, J., \& López-Mascaraque, L. (2016). UbC-StarTrack, a clonal method to target the entire progeny of individual progenitors. Scientific Reports, 6, 33896. https://doi.org/10. 1038/srep33896

García-Marqués, J., \& López-Mascaraque, L. (2013). Clonal identity determines astrocyte cortical heterogeneity. Cerebral Cortex, 23(6), 1463-1472. https://doi.org/10.1093/cercor/bhs134

García-Marqués, J., \& López-Mascaraque, L. (2017). Clonal mapping of astrocytes in the olfactory bulb and rostral migratory stream. Cerebral Cortex, 27(3), 2195-2209. https://doi.org/10.1093/cercor/bhw071

Giaume, C., Koulakoff, A., Roux, L., Holcman, D., \& Rouach, N. (2010). Astroglial networks: A step further in neuroglial and gliovascular interactions. Nature Reviews. Neuroscience, 11(2), 87-99. https://doi.org/ 10.1038/nrn2757

Giaume, C., Orellana, J. A., Abudara, V., \& Sáez, J. C. (2012). Connexinbased channels in astrocytes: How to study their properties. Methods 
in Molecular Biology, 814, 283-303. https://doi.org/10.1007/978-161779-452-0_19

Hochstim, C., Deneen, B., Lukaszewicz, A., Zhou, Q., \& Anderson, D. J. (2008). Identification of positionally distinct astrocyte subtypes whose identities are specified by a homeodomain code. Cell, 133, 510-522. https://doi.org/10.1016/j.cell.2008.02.046

Höfer, T., Venance, L., \& Giaume, C. (2002). Control and plasticity of intercellular calcium waves in astrocytes: A modeling approach. The Journal of Neuroscience: The Official Journal of the Society for Neuroscience, 22 (12), 4850-4859.

Houades, V., Koulakoff, A., Ezan, P., Seif, I., \& Giaume, C. (2008). Gap junction-mediated astrocytic networks in the mouse barrel cortex. The Journal of Neuroscience: The Official Journal of the Society for Neuroscience, 28(20), 5207-5217. https://doi.org/10.1523/JNEUROSCI.510007.2008

Houades, V., Rouach, N., Ezan, P., Kirchhoff, F., Koulakoff, A., \& Giaume, C. (2006). Shapes of astrocyte networks in the juvenile brain. Neuron Glia Biology, 2(1), 3-14. https://doi.org/10.1017/ S1740925X06000081

Kuga, N., Sasaki, T., Takahara, Y., Matsuki, N., \& Ikegaya, Y. (2011). Largescale calcium waves traveling through astrocytic networks in vivo. The Journal of Neuroscience: The Official Journal of the Society for Neuroscience, 31(7), 2607-2614. https://doi.org/10.1523/JNEUROSCI.531910.2011

Martín-López, E., García-Marques, J., Núñez-Llaves, R., \& LópezMascaraque, L. (2013). Clonal astrocytic response to cortical injury. PLoS ONE, 8, e74039. https://doi.org/10.1371/journal.pone.007 4039

Nagy, J. I., \& Rash, J. E. (2000). Connexins and gap junctions of astrocytes and oligodendrocytes in the CNS. Brain Research Reviews, 32(1), 29-44.

Oberheim, N. A., Goldman, S. A., \& Nedergaard, M. (2012). Heterogeneity of astrocytic form and function. Methods in Molecular Biology, 814, 23-45. https://doi.org/10.1007/978-1-61779-452-0_3

Ogata, K., \& Kosaka, T. (2002). Structural and quantitative analysis of astrocytes in the mouse hippocampus. Neuroscience, 113(1), 221-233.

Orthmann-Murphy, J. L., Abrams, C. K., \& Scherer, S. S. (2008). Gap junctions couple astrocytes and oligodendrocytes. Journal of Molecular Neuroscience, 35(1), 101-116. https://doi.org/10.1007/s12031-0079027-5

Pannasch, U., \& Rouach, N. (2013). Emerging role for astroglial networks in information processing: From synapse to behavior. Trends in Neurosciences, 36(7), 405-417. https://doi.org/10.1016/j.tins.2013.04.004
Perea, G., \& Araque, A. (2007). Astrocytes potentiate transmitter release at single hippocampal synapses. Science, 317(5841), 1083-1086.

R Core Team6. (2018). R: A language and environment for statistical computing. Vienna, Austria: R Foundation for Statistical Computing. https:// www.R-project.org/

Rouach, N., Koulakoff, A., Abudara, V., Willecke, K., \& Giaume, C. (2008). Astroglial metabolic networks sustain hippocampal synaptic transmission. Science, 322(5907), 1551-1555. https://doi.org/10.1126/science.1164022

Roux, L., Benchenane, K., Rothstein, J. D., Bonvento, G., \& Giaume, C. (2011). Plasticity of astroglial networks in olfactory glomeruli. Proceedings of the National Academy of Sciences of the United States of America, 108(45), 18442-18446. https://doi.org/10.1073/pnas.1107386108

Sasaki, T., Kuga, N., Namiki, S., Matsuki, N., \& Ikegaya, Y. (2011). Locally synchronized astrocytes. Cerebral Cortex, 21(8), 1889-1900. https:// doi.org/10.1093/cercor/bhq256

Scemes, E., \& Giaume, C. (2006). Astrocyte calcium waves: What they are and what they do. Glia, 54(7), 716-725.

Theis, M., \& Giaume, C. (2012). Connexin-based intercellular communication and astrocyte heterogeneity. Brain Research, 1487, 88-98. https://doi.org/10.1016/j.brainres.2012.06.045

Verkhratsky, A., \& Nedergaard, M. (2018). Phisiology of astroglia. Physiological Reviews, 98(1), 239-389. https://doi.org/10.1152/physrev.00042.2016

Ye, Z. C., Wyeth, M. S., Baltan-Tekkok, S., \& Ransom, B. R. (2003). Functional hemichannels in astrocytes: A novel mechanism of glutamate release. The Journal of Neuroscience, 23(9), 3588-3596.

Yu, Y. C., He, S., Chen, S., Fu, Y., Brown, K. N., Yao, X. H., ... Shi, S. H. (2012). Preferential electrical coupling regulates neocortical lineage-dependent microcircuit assembly. Nature, 486(7401), 113-117. https://doi.org/10. 1038/nature10958

\section{SUPPORTING INFORMATION}

Additional supporting information may be found online in the Supporting Information section at the end of this article.

How to cite this article: Gutiérrez Y, García-Marques J, Liu X, et al. Sibling astrocytes share preferential coupling via gap junctions. Glia. 2019;1-7. https://doi.org/10.1002/glia.23662 